\title{
Correction of rigid body motion in deformation measurement of rotating objects
}

Pedro J. Sousa ${ }^{\mathrm{a}, \mathrm{b}, *}$, João Manuel R. S. Tavares ${ }^{\mathrm{a}, \mathrm{b}}$, Paulo J. S. Tavares ${ }^{\mathrm{a}}$, Pedro M. G. P. Moreira ${ }^{a}$

${ }^{a}$ Instituto de Ciência e Inovação em Engenharia Mecânica e Engenharia Industrial, Universidade do Porto, Rua Dr. Roberto Frias, s/n, 4200-465 Porto, Portugal

${ }^{b}$ Faculdade de Engenharia, Universidade do Porto, Rua Dr. Roberto Frias, 400, 4200-465 Porto, Portugal

\begin{abstract}
When using image-based measurement solutions for monitoring the displacement of rotating objects, it is necessary to trigger the image acquisition system at precise positions. Triggering errors lead to artificial rigid motions in the output that are hard to remove independently. Thus, a methodology was developed to enable the removal of these errors while maintaining the true rotations, such as those relating to the angle of attack.

The proposed methodology involves detecting an object's axis and centre of rotation and then using that knowledge to correct acquired deformation data. Additionally, it also enables the representation of displacements in a more representative coordinate system, i.e., one that includes the rotation axis.

While common solutions for this problem use either manually positioned references or track a single-point's rotation, the proposed methodology is based on a least-squares approach. This results in a more accurate measurement of the rotation axis and, thus, improves the quality of the results.

The methodology was experimentally validated and it was shown that its implementation is accurate, with errors below $3 \%$. Additionally, it was applied to an actual experimental situation and the results were compared to the uncorrected data, while highlighting the most relevant improvements.
\end{abstract}

Keywords: Digital Image Correlation, Helicopter blade deformation, Image processing, Point cloud processing

\section{Introduction}

Rotating structures are commonly used for a wide range of applications, which makes their motion study important for the improvement of current solutions [1], particularly in aeronautics where most rotating objects are long and slender, exhibiting considerable deformation [2]. There are several methods to study the deformation of such objects, either with or without contact, for example using strain gauges or digital image correlation [3, 4]. Image-based methods have a number of advantages over traditional methods, such as being

\footnotetext{
*Corresponding author

Email address: p.sousa@fe.up.pt (Pedro J. Sousa)
} 
non-contact or able to acquire full displacement or deformation fields, as opposed to point-to-point techniques [5]. As such, their use does not influence the movement/deformation of the object under study, which is important in many applications $[3,6]$.

In recent years, image methods have been used to perform measurements in rotating objects, ranging from shafts [7] to propellers [6, 8] and wind turbines [9]. Many of these works, such as $[6,3,10,11,12,13,9,14,15,8,16,17]$, involve the acquisition of shape and displacements using 3D digital image correlation.

The alignment of the world coordinates with the rotation axis is crucial for displacement measurements $[13,18,17]$, in that if the coordinate systems are misaligned, the comparatively large deformations along the rotation axis also contribute towards measurements along other axes. As such, this problem is often tackled by defining the rotation axis [18] and creating a new coordinate system, intrinsic to the motion. Sicard and Sirohi approached this by carefully aligning a calibration plate with the rotor hub plane and defining the coordinate system from this plate [13]. On the other hand, Winstroth et al., proposed a method where one data point from the tip of the rotor is extracted for an entire rotation, which would then be used to define a $3 \mathrm{D}$ circle using the least-squares method, defining the centre of rotation [18]. Other works involving rotating objects often do not approach this problem because the target of their research can be obtained even with misaligned axis. For example, Rizo-Patron and Sirohi were able to perform modal analysis $[15,8]$, and obtain the natural frequencies and mode shapes, without considering misalignments, as it had no effect on these results.

Another important factor when performing measurements in rotating objects is that the camera should be precisely synchronized with the rotation to record the target object always in the same position [6]. However, in [16, 17], it was noted that when using a static reference, its positioning is also important, as misalignments between the acquired images in stationary and the rotating situations will cause rigid-body rotations that may hide the sought-after deformations. As such, they should be avoided or removed prior to the analysis, because they only represent a different angular position and not an actual displacement.

Commercial Digital Image Correlation (DIC) software usually feature rigid body motion removal features that can remove rotations $[19,20]$ in order to find only the deformation components of displacement [19]. For example, using VIC-3D from Correlated Solutions, there are three rigid body motion removal approaches [19]: average transformation, keeping three fixed points or just one fixed point. While the latter will not affect rotations, maintaining the original values, with the other two all displacements and rotations will be relative to either the average transformation or the three selected points. This means that to remove rotation, it is necessary to do it along all axes when using that software.

Winstroth and Seume suggest, as a final remark in [18], that one way to remove these rotations could be to realign the deformed point clouds with the reference one by minimizing the distance between them close to the blade hub. Additionally, this could also be approached using a relative technique, where the reference image is also acquired during rotation, as suggested by Stasicki and Boden [6] but with a different goal, which was that of reducing motion blur effects. 
The proposed methodology is aimed at tackling simultaneously the aforementioned two problems, by defining the rotation axis using two reference situations in different angular positions and using this knowledge to align every dynamically loaded situations to one of these references, where a projection of the blade perpendicular to the rotation axis is the matching target. This enables the removal of rigid rotations that do not impact the system's function while maintaining the others, of which the attack angle is the most important example. Afterwards, that same information is used to represent the results in the new coordinate system.

In this article, a novel methodology is proposed. Then, two implemented test procedures are described. The first one deals with synthetic data and attempts to recover the parameters that were used to generate it. The other is an experimental validation procedure, where known displacements were imposed and the proposed methodology was used in order to accurately measure them. Finally, an example application is presented and the results obtained from the proposed methodology are compared with the uncorrected ones and a critical discussion is presented.

\section{Proposed Methodology}

A novel methodology was developed to detect an object's axis and centre of rotation and use that information to correct acquired deformation data from a rotating object.

For this, an experimental setup that is similar to the one used in [17] and shown schematically in Figure 1 was considered. It is used to measure the displacements of rotating objects, such as helicopter blades and requires that the region of interest is painted with a random speckle pattern, as the blade in the right side of the schematic.

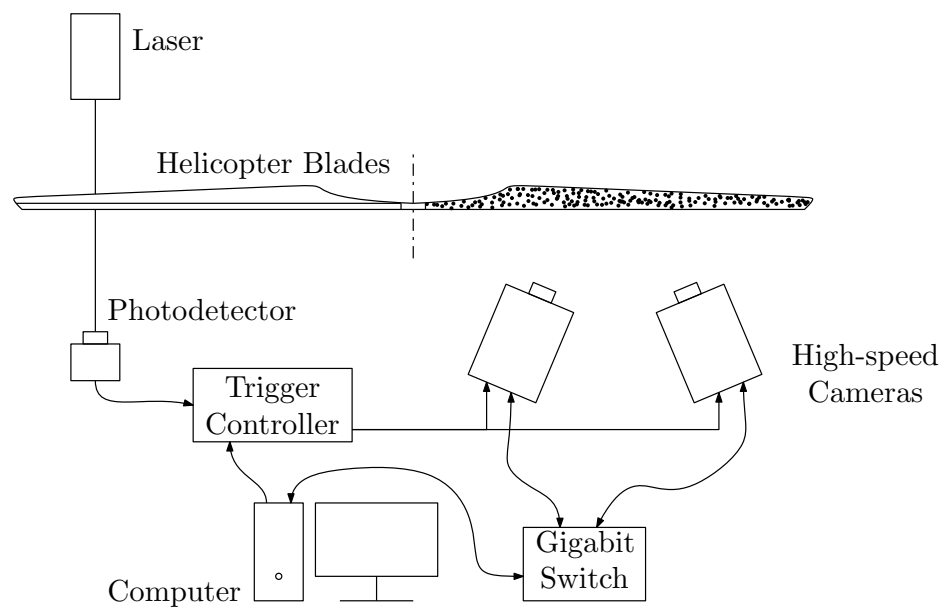

Figure 1: Schematic of the experimental setup

In order to measure deformation using 3D Digital Image Correlation (DIC), it is necessary to, first, calibrate the stereo camera system using images of a known pattern and, then, acquire a pair of stereo images to define the reference situation [21]. Afterwards, while target is rotating the blades will periodically 
interrupt a laser beam, which generates a signal on the photodetector's output. This is then processed by the controller to trigger the high-speed cameras and simultaneously acquire stereo images of the rotating object in a particular position in space. The displacements are then obtained by comparing the reference and deformed situations [21].

The proposed methodology aims at: removing any rigid rotations that are created by misalignments between the reference and deformed situations; and aligning the world coordinates with the rotation axis. Its main steps include:

- Acquisition of reference images for calibration of the rotation axis, in two different angular positions;

- Acquisition of images under dynamic loading conditions;

- Image processing using Digital Image Correlation [21, 22], that includes the removal of outliers that are created in regions with poor or no speckle pattern or with other correlation issues, such as motion blurred or out-offocus regions;

- Calculation of the rotation axis from the reference images' data;

- Projection of the data from the situations with loading in a plane normal to the rotation axis;

- Calculation of the best-fit rotation angles between these situations and a reference one;

- Correct the point clouds using the calculated rotations;

- Calculate displacements and other interest parameters.

These steps are shown schematically in Figure 2.

The images are initially acquired and then processed with the DIC software package VIC-3D 2012 from U.S.A.'s Correlated Solutions. The resulting point clouds are exported and corrected according to the following workflow.

\subsection{Calculation of the centre and axis of rotation}

The first step of the methodology is to calculate the best-fit rotation axis, using the Least-Squares method developed by Spoor and Veldpaus [23, 24] and taking into account Rose and Richards' suggestions [25].

Two point clouds at different angular positions of the object are used. In order to reduce errors, a large angle between them is advantageous and the reference images should have only undergone rotation along the target axis. Defining the points in the first point cloud as $a_{i}$ and the ones in the second point cloud as $p_{i}$, it is possible to define one matrix for each point cloud:

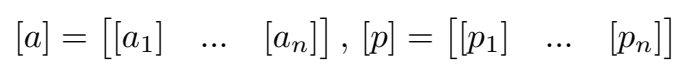

The centroids of both sets, $\bar{a}$ and $\bar{p}$, can be calculated simply as an average of each coordinate.

An auxiliary matrix, $M$, can then be calculated as:

$$
M=\frac{1}{n}[a] \cdot[p]^{T}-\bar{a} \cdot \bar{p}^{T}
$$




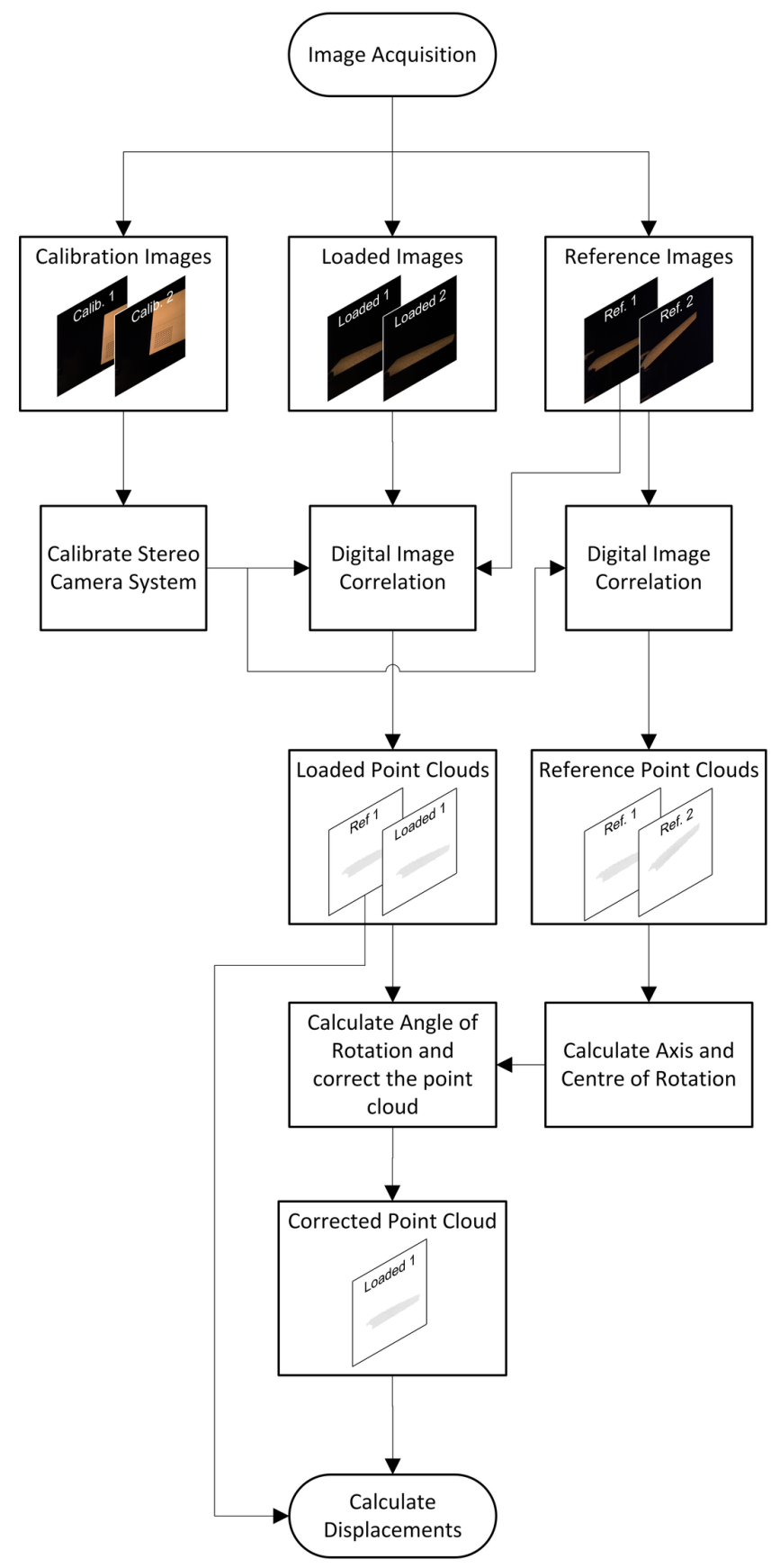

Figure 2: Proposed acquisition and measurement methodology 
Matrix $M$ can be used to calculate a symmetric matrix of Lagrangian multipliers as $S^{2}=M^{T} \cdot M$ [23]. From the definition of eigenvalues and eigenvectors, it is seen that $M^{T} M=V D^{2} V^{T}$, where the eigenvectors, $V_{i}$, are the columns of matrix $V$ and the eigenvalues, $d_{i}$, are the positive square roots of the diagonal of $D^{2}$ :

$$
D^{2}=\left[\begin{array}{ccc}
d_{1}^{2} & 0 & 0 \\
0 & d_{2}^{2} & 0 \\
0 & 0 & d_{3}^{2}
\end{array}\right]
$$

The parameters in (2) are calculated according to Spoor and Veldpaus' analysis [23] towards the minimization of an overall measure for the difference between the two point clouds.

The rotation matrix is calculated as in [25] by following the appropriate branch of:

$$
\left\{\begin{array}{lll}
\text { If } V_{2} \times V_{3}=+V_{1}, \text { then } & R=\left[\begin{array}{lll}
\frac{m_{2} \times m_{3}}{d_{2} \cdot d_{3}} & \frac{m_{2}}{d_{2}} & \frac{m_{3}}{d_{3}}
\end{array}\right] \cdot V^{T} \\
\text { If } V_{2} \times V_{3}=-V_{1}, \text { then } & R=\left[\begin{array}{lll}
-\frac{m_{2} \times m_{3}}{d_{2} \cdot d_{3}} & \frac{m_{2}}{d_{2}} & \frac{m_{3}}{d_{3}}
\end{array}\right] \cdot V^{T}
\end{array}\right.
$$

where $m_{i}$ are the columns of:

$$
m=\left[\begin{array}{lll}
m_{1} & m_{2} & m_{3}
\end{array}\right]=M \cdot V
$$

After the application of Spoor and Veldpaus' approach, it is then necessary to calculate the centre of rotation.

There are two possible representations of a rotation matrix [26, 27], following the logic of object or axis rotation, whose relationship is $R_{\text {object }}=R_{\text {axis }}^{T}$. Therefore, the result from Eq. 4 can be transposed to match the intended representation.

From matrix $R$, it is trivial to obtain the axis of rotation, $\vec{u}$. However, in order to fully characterize the rotation, a point contained in this axis is also necessary. Thus, the centre of rotation, $x_{0}$, is calculated by considering that the relationship between $\bar{a}$ and $\bar{p}$ is:

$$
\bar{p}=R \cdot\left(\bar{a}-x_{0}\right)+x_{0}
$$

What means that the centre of rotation can then be calculated as:

$$
x_{0}=(R-I) \backslash(R \cdot \bar{a}-\bar{p})
$$

where $I$ is the $\mathbb{R}^{3}$ identity matrix

Since any point that belongs to the axis will verify Eq. 6, the system of equations will have multiple solutions and the matrix $(R-I)$ will usually be close to singular, which can lead to incorrect results with usual linear solvers. As such, possible alternative solving methodologies include the use of a minimum norm least-squares solution through Complete Orthogonal Decomposition [28, 29] or the use of the Moore-Penrose pseudoinverse, $(R-I)^{+}$, which uses Singular Value Decomposition $[29,30]$. It is important to note that both of these options should provide accurate results, but not exactly the same point. The first approach is also generally regarded as more efficient [28].

Having obtained one point in the axis, it is possible to obtain any other. For the problem under study, a good choice of centre of rotation can be one that has $z\left(x_{0}\right)=0$, which can be calculated as: 


$$
x_{0_{\text {ref }}}=x_{0}-\vec{u} \cdot \frac{z\left(x_{0}\right)}{z(\vec{u})}
$$

where $z\left(x_{0}\right)$ is the $\mathrm{Z}$ coordinate of $x_{0}$ and $z(\vec{u})$ is the component of $\vec{u}$ along $\mathrm{Z}$.

\subsection{Correction of the Point Clouds}

In order to fully characterize the necessary movement to correct the point clouds, there are three essential parameters: the axis, the centre of rotation and the angle. The first two are previously obtained from the calibration procedure. As such, using these parameters, the rotation angle is then calculated from each measurement through a least-squares fit in a projection plane normal to the axis of rotation. This allows to get the best-fit angle of rotation even with significant deformations in other axes.

When defining that projection plane, it was intended to maintain an axis as closely aligned with the original $\mathrm{X}$ axis as possible. As such, the axes $\overrightarrow{v_{1}}$ and $\overrightarrow{v_{2}}$ are calculated as:

$$
\begin{gathered}
\overrightarrow{v_{2}}=\vec{u} \times\left[\begin{array}{l}
1 \\
0 \\
0
\end{array}\right] \\
\overrightarrow{v_{1}}=\overrightarrow{v_{2}} \times \vec{u}
\end{gathered}
$$

After dividing each new vector by its norm, the new orthonormal base, $\left(\overrightarrow{v_{1}}, \overrightarrow{v_{2}}, \vec{u}\right)$, is defined.

To calculate the angle of rotation, $b_{i}$ are defined as the reference situation points and $q_{i}$ as the ones with dynamic load. Here, the reference set of points, $b_{i}$, could be the same as one of the sets used in Section 2.1, $a_{i}$ or $p_{i}$.

It should be noted that it is not necessary to use the total amount of points for this registration. It is also possible to select one edge or other notable feature as the registration target.

Defining $\vec{x}_{1 i}$ as the smaller norm vector from the rotation axis to a particular reference point $b_{i}$ yields:

$$
\vec{x}_{1 i}=b_{i}-e_{i}
$$

where $e_{i}$ is the rotation axis' point closest to $b_{i}$. It can be calculated as [31]:

$$
e_{i}=x_{0}+t_{i} \cdot \vec{u}
$$

where $x_{0}$ is the previously calculated rotation centre and $t_{i}$ is:

$$
t_{i}=-\frac{\left(x_{0}-b_{i}\right) \cdot \vec{u}}{\|\vec{u}\|^{2}}
$$

Next, the projection of $\vec{x}_{1 i}$ along both $\overrightarrow{v_{1}}$ and $\overrightarrow{v_{2}}$ [32] is obtained by:

$$
\begin{aligned}
& \left.{\overrightarrow{x_{1}}}_{i}\right|_{v_{1}}=\frac{\overrightarrow{v_{1}} \cdot \overrightarrow{x_{1 i}}}{\left\|\overrightarrow{v_{1}}\right\|} \\
& {\overrightarrow{x_{1}}}_{i_{v_{2}}}=\frac{\overrightarrow{v_{2}} \cdot \overrightarrow{x_{1}}}{\left\|\overrightarrow{v_{2}}\right\|}
\end{aligned}
$$


If the base is orthonormal, the norm of $\overrightarrow{v_{1}}$ and $\overrightarrow{v_{2}}$ will be 1 (one) and, as such, do not have to be considered.

Finally, the point projected in this plane will be:

$$
\left.b_{i}\right|_{v_{1}, v_{2}}=\left[\begin{array}{c}
\left.\overrightarrow{x_{1}}\right|_{v_{1}} \\
\left.\vec{x}_{1}\right|_{v_{2}} \\
0
\end{array}\right]
$$

With the due considerations, this can be extended to the remaining points, $q_{i}$, in order to obtain the projection:

$$
\left.q_{i}\right|_{v_{1}, v_{2}}=\left[\begin{array}{c}
\left.\overrightarrow{x_{2}} i\right|_{v_{1}} \\
\left.\overrightarrow{x_{2}} i\right|_{v_{2}} \\
0
\end{array}\right]
$$

where $\vec{x}_{2}$ is the smallest norm vector from the rotation axis to a particular point $q_{i}$.

Following the notation used in Eq. 1, the projected points can be defined as:

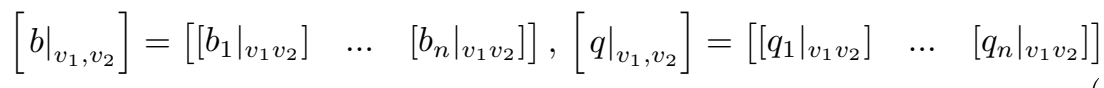

The angle is then calculated by applying Spoor and Valdepaus' algorithm until Eq. 4, replacing the matrices $[a]$ and $[p]$ with $\left[\left.b\right|_{v_{1}, v_{2}}\right]$ and $\left[\left.q\right|_{v_{1}, v_{2}}\right]$. This again returns a rotation matrix, from where an angle, $\alpha$, and a new axis can be extracted.

If the orthogonal base was not created using Eqs. 9 and 10 it may be necessary to correct the angle's signal, $\alpha=s \cdot \alpha$, where:

$$
s=\vec{u} \cdot\left(\overrightarrow{v_{1}} \times \overrightarrow{v_{2}}\right)
$$

Additionally, the axis extracted from the last rotation matrix can be either $\left[\begin{array}{lll}0 & 0 & 1\end{array}\right]$ or $\left[\begin{array}{lll}0 & 0 & -1\end{array}\right]$. In the first case, the angle has to be corrected as:

$$
\alpha=-\alpha
$$

From this angle $\alpha$ and the $\vec{u}$ axis, a new rotation matrix is created, $R_{C}$, and is then used to correct the dynamic load situation points $[q]$ :

$$
[Q]=R_{C} \cdot\left([q]-\left[X_{0}\right]\right)+\left[X_{0}\right]
$$

where $\left[X_{0}\right]$ is a matrix of the same size as $[q]$, where in every column are the coordinates of the centre of rotation:

$$
\left[X_{0}\right]=\left[\begin{array}{lll}
{\left[x_{0}\right]} & \ldots & {\left[x_{0}\right.}
\end{array}\right]
$$

The application of this approach is schematically shown in Figure 3. 


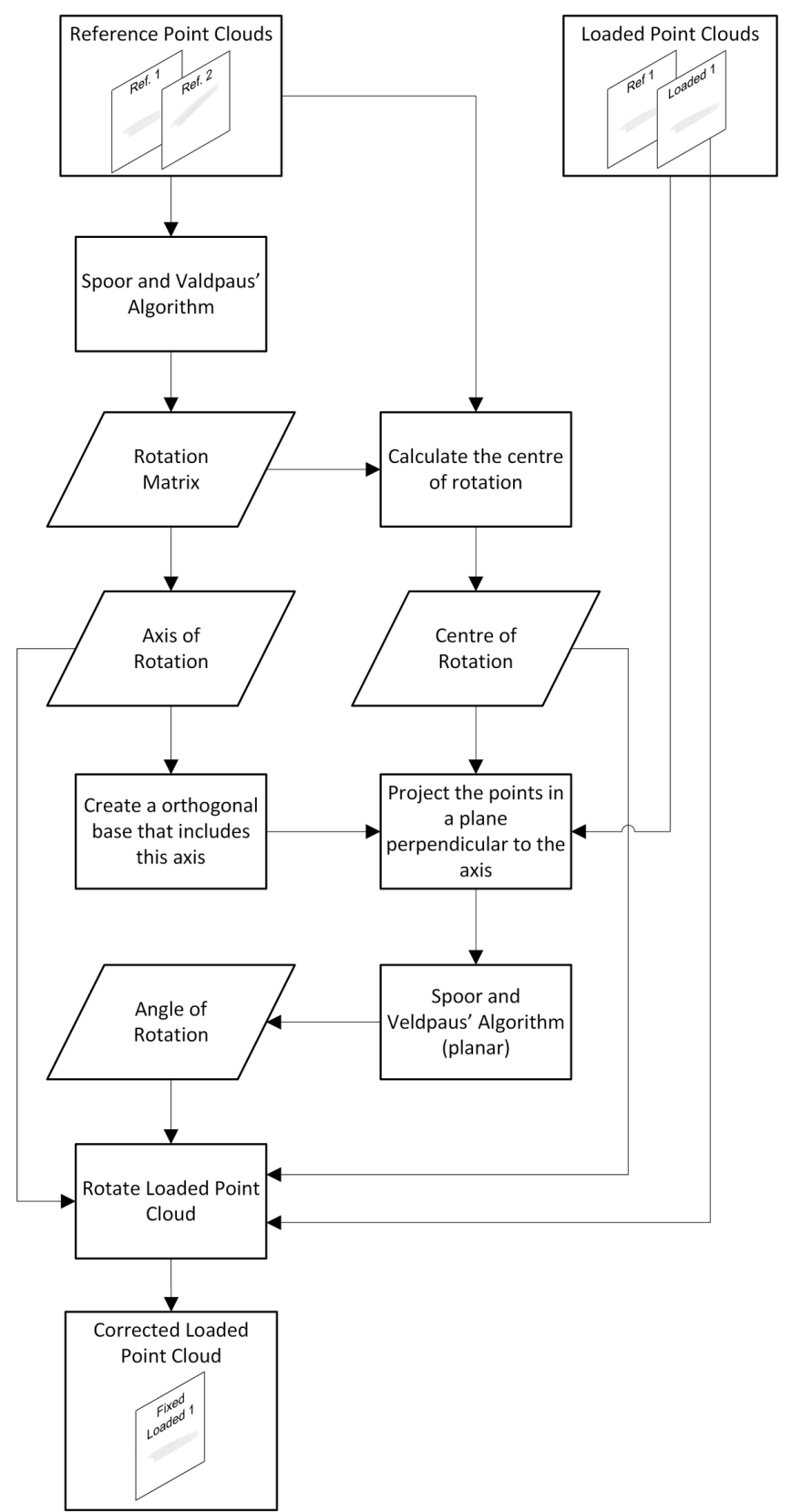

Figure 3: Approach developed for point cloud correction 


\subsection{Calculation of the Displacements}

Finally, it is possible to calculate the displacements in the $\left(\overrightarrow{v_{1}}, \overrightarrow{v_{2}}, \vec{u}\right)$ coordinate system, enabling a more intuitive presentation of the acquired data. For this, it is necessary to define each point in the new coordinate system. This can be achieved by applying Eqs. 11 to 17 and considering the result of Eq. 13 as the third component of Eqs. 16 and 17:

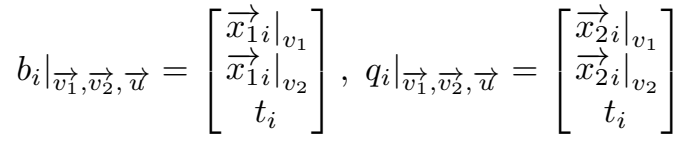

Then, displacements in the new coordinate system can be directly calculated from the coordinates of matching points in both sets.

\section{Tests and discussion}

In order to ensure that the proposed methodology gives good results for practical application, it is necessary to test its behaviour. As such, two complementary approaches were used: numerical and experimental. The first one involves the use of synthetic data to test the applicability of the methodology in identifying movement parameters. The second one was performed by imposing known displacements on a polymer blade and measuring them using the proposed methodology. The obtained displacements were then compared with the imposed ones in order to validate the results. For each approach, a brief discussion regarding the obtained errors is included.

\subsection{Synthetic data}

A set of 5 (five) points with coordinates in the $[0,10]$ range was defined through a pseudo-random function, which led to:

$$
[a]=\left[\begin{array}{lllll}
9.0579 & 1.2699 & 9.1338 & 6.3236 & 0.9754 \\
2.7850 & 5.4688 & 9.5751 & 9.6489 & 1.5761 \\
9.7059 & 9.5717 & 4.8538 & 8.0028 & 1.4189
\end{array}\right]
$$

Several test scenarios were then defined by combining a rotation axis, an angle and a centre of rotation as indicated in Table 1.

\begin{tabular}{|c|c|c|c|}
\hline & $\vec{u}$ & $\alpha$ & $x_{0}$ \\
\hline \multirow{3}{*}{1} & {$[0]$} & \multirow{3}{*}{$40^{\circ}$} & {$[0.9]$} \\
\hline & 0 & & 0.9 \\
\hline & 1 & & 0.9 \\
\hline \multirow{3}{*}{2} & {$[-0.3333$} & \multirow{3}{*}{$151.8341^{\circ}$} & {$[6.5574]$} \\
\hline & 0.6667 & & 0.3571 \\
\hline & 0.6667 & & 8.4913 \\
\hline
\end{tabular}

Table 1: Parameters that were combined to define each test scenario

Every possible combination of the three parameters in Table $1\left(\vec{u}_{i}, \alpha_{j}\right.$ and $x_{0 k}$ ) was tested. As such, a rotation of $\alpha_{j}$ degrees around axis $\vec{u}_{i}$ and centre $x_{0 k}$ 
(input values) was applied to the set of random points $[a]$. Then, the developed algorithm was applied to recover an angle and an axis defined by a normal vector and a centre of rotation (output values) from the generated points.

Without forcing the third component of $x_{0}$ to 0 (zero), the calculation of the output centre of rotation is underdetermined and, as such, it can be any point in the axis as long as it satisfies:

$$
x_{0_{\text {output }}}=x_{0_{\text {input }}}+t \cdot \vec{u}
$$

where $t \in \mathbb{R}$.

The tested combinations as well as the obtained results, including the value of $t$ required to verify Eq. 25, are indicated in Table 2 .

Table 2: Obtained results after application of the algorithm to the synthetic data sets

\begin{tabular}{|c|c|c|c|c|}
\hline \multirow{2}{*}{$\begin{array}{c}\text { Input } \\
\left(\vec{u}, \alpha, x_{0}\right)\end{array}$} & \multicolumn{4}{|c|}{ Output } \\
\hline & Normal vector & Angle & Rotation centre & $\mathrm{t}$ \\
\hline \multirow{3}{*}{$\vec{u}_{1}, \alpha_{1}, x_{0_{1}}$} & {$[0.0000$} & & 0.9000 & \\
\hline & 0.0000 & 40.0000 & 0.9000 & -0.9000 \\
\hline & 1.0000 & & {$[-0.0000]$} & \\
\hline \multirow{3}{*}{$\vec{u}_{1}, \alpha_{1}, x_{0_{2}}$} & {$[0.0000]$} & \multirow{3}{*}{40.0000} & 6.5574 & \multirow{3}{*}{-8.4913} \\
\hline & 0.0000 & & 0.3571 & \\
\hline & 1.0000 & & -0.0000 & \\
\hline \multirow{3}{*}{$\vec{u}_{1}, \alpha_{2}, x_{0_{1}}$} & 0.0000 & \multirow{3}{*}{151.8341} & {$[0.9000$} & \multirow{3}{*}{-0.9000} \\
\hline & -0.0000 & & 0.9000 & \\
\hline & 1.0000 & & 0.0000 & \\
\hline \multirow{3}{*}{$\vec{u}_{1}, \alpha_{2}, x_{0_{2}}$} & -0.0000 & \multirow{3}{*}{151.8341} & 6.5574 & \multirow{3}{*}{-8.4913} \\
\hline & 0.0000 & & 0.3571 & \\
\hline & 1.0000 & & 0.0000 & \\
\hline \multirow{3}{*}{$\vec{u}_{2}, \alpha_{1}, x_{0_{1}}$} & -0.3333 & \multirow{3}{*}{40.0000} & {$[1.2000$} & \multirow{3}{*}{-0.9000} \\
\hline & 0.6667 & & 0.3000 & \\
\hline & 0.6667 & & 0.3000 & \\
\hline \multirow{3}{*}{$\vec{u}_{2}, \alpha_{1}, x_{0_{2}}$} & -0.3333 & \multirow{3}{*}{40.0000} & 5.9396 & \multirow{3}{*}{1.8533} \\
\hline & 0.6667 & & 1.5926 & \\
\hline & 0.6667 & & 9.7268 & \\
\hline \multirow{3}{*}{$\vec{u}_{2}, \alpha_{2}, x_{0_{1}}$} & -0.3333 & \multirow{3}{*}{151.8341} & {$[1.2000$} & \multirow{3}{*}{-0.9000} \\
\hline & 0.6667 & & 0.3000 & \\
\hline & 0.6667 & & 0.3000 & \\
\hline \multirow{3}{*}{$\vec{u}_{2}, \alpha_{2}, x_{0_{2}}$} & {$[-0.3333]$} & \multirow{3}{*}{151.8341} & 7.7951 & \multirow{3}{*}{-3.7131} \\
\hline & 0.6667 & & -2.1183 & \\
\hline & 0.6667 & & 6.0159 & \\
\hline
\end{tabular}

While it is easily perceived from Table 2 that the obtained normal vectors and angles are all accurate at least to the fourth decimal place, the output 
centres of rotation are related to the input values through Eq. 25. A more detailed presentation of the obtained errors is presented in Table 3. Here, the centre of rotation error was calculated after application of Eq. 25 and any error values of 0 (zero) should be understood as being inferior to the machine eplison (eps), i.e. the minimum difference between two floating-point double-precision numbers.

Table 3: Errors obtained from the application of the algorithm to the synthetic data sets

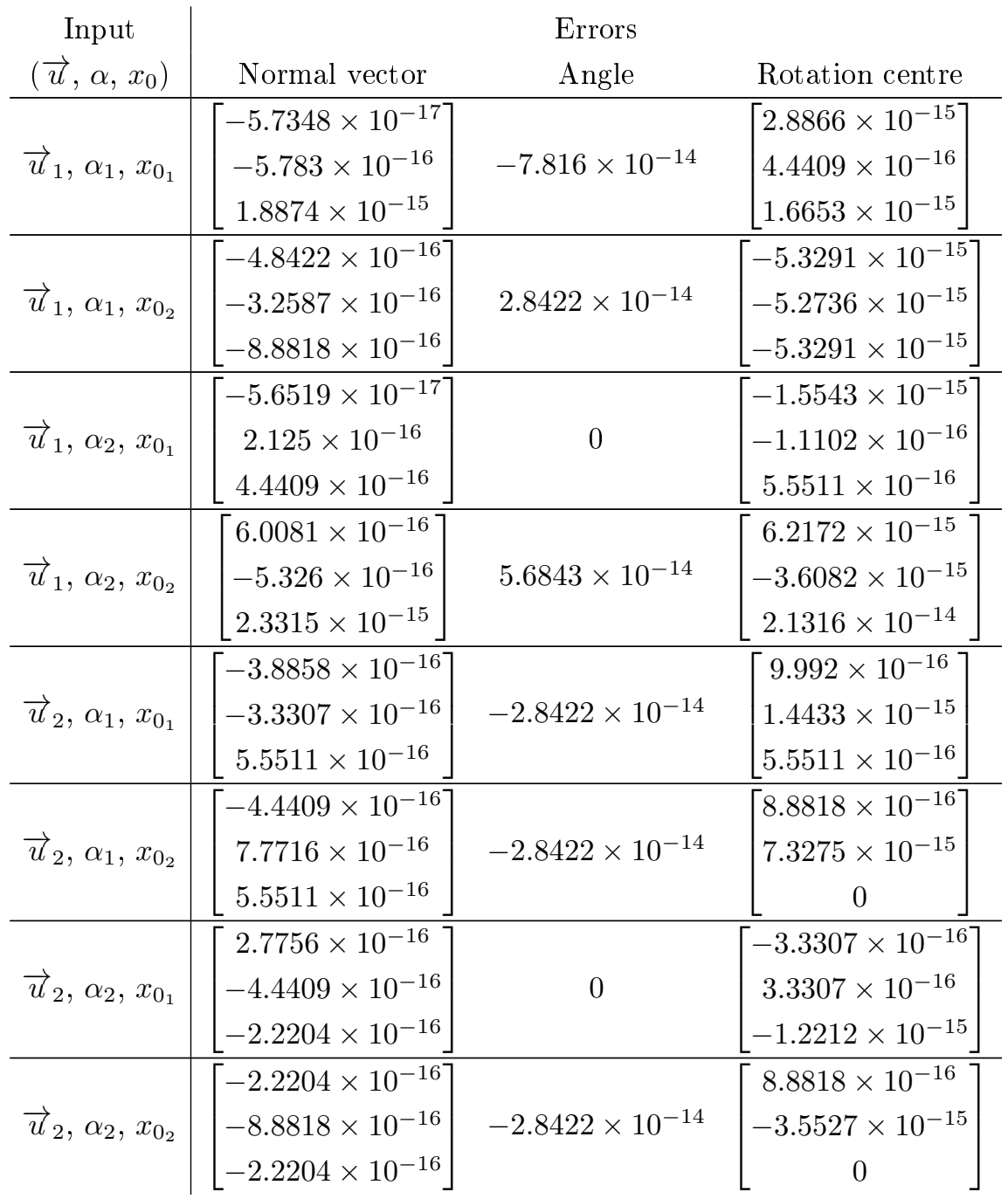

Since all of the calculated errors are below $10^{-13}$, it is appropriate to consider that the proposed methodology is capable of obtaining accurate results.

\subsection{Experimental Validation}

To complement the previous analysis, an experimental validation procedure was devised. A manual linear stage rigidly connected to a $12 \mathrm{~mm}$ diameter metallic rod was used to impose and measure displacements. Due to the size and 
material of the rod, it is expected that its deformation is neglectable compared to the polymeric blade.

To define the reference point from which the displacements applied to the manual stage are also applied to the target, an external methodology based in Projection Moiré [33] was used. This involved the use of a fringe projector and a camera to compare the position of the projected grid in different points in time by subtracting the images and analysing the generated fringe pattern. These, along with the remaining components of the setup, are shown in Figures 4 and 5 . Even though the experimental validation was performed statically, this setup was designed for dynamic usage $[16,17]$. Here, the laser and photodetector were used to generate a signal from the rotation of the blades that was then processed by the controller to trigger both high-speed cameras simultaneously at a particular location. Besides this, the controller is also capable of generating trigger signals independently of the input, enabling manual simultaneous image acquisition.

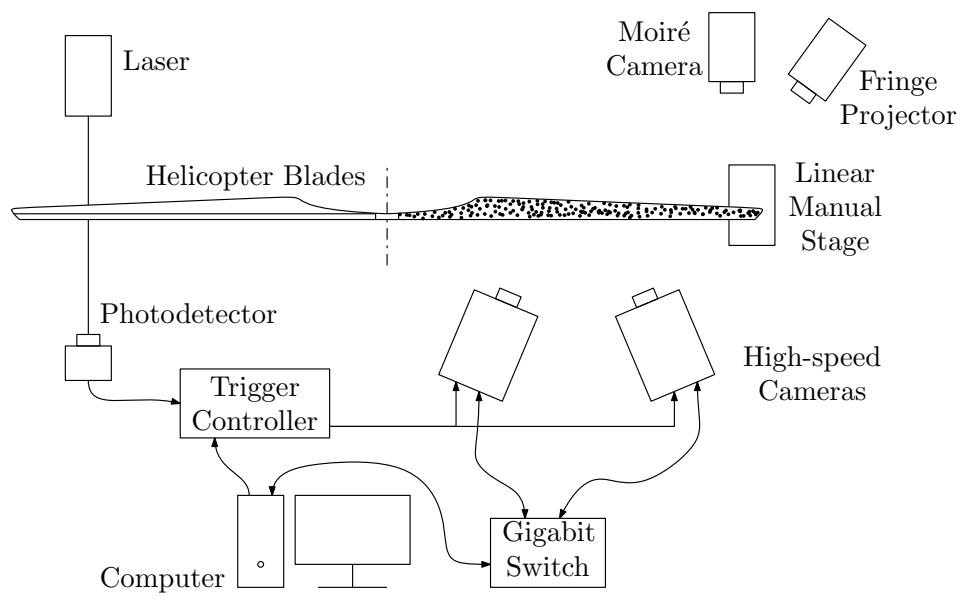

Figure 4: Schematic of the experimental setup used for validation

The required images were then obtained. Bundle calibration was performed by placing a pattern in multiple orientations inside the work area and the calibration images were acquired.

Reference images were then obtained by manually positioning the RC helicopter's blade in two angular positions, with a metallic part keeping it rigidly connected to the rotation axis.

Before imposing displacements to the blade, the Projection Moiré system was used to position the linear manual stage at the point where fringes start appearing.

From previous works, the displacements that were measured for rotations of around $680 \mathrm{rpm}$ along the Z-axis were close to $6 \mathrm{~mm}$ [17]. Considering that this is smaller than the real value, a set of four displacements, 2.0, 4.0, 5.0 and $7.0 \mathrm{~mm}$, were imposed and images were acquired for each. Here, the maximum displacement matches the expected real value at $680 \mathrm{rpm}$ and there are three additional not evenly spaced points at integer values. The acquired images were processed by Correlated Solutions' VIC-3D and exported to MATLAB for processing. Applying the developed algorithm, the axis and centre of rotation 


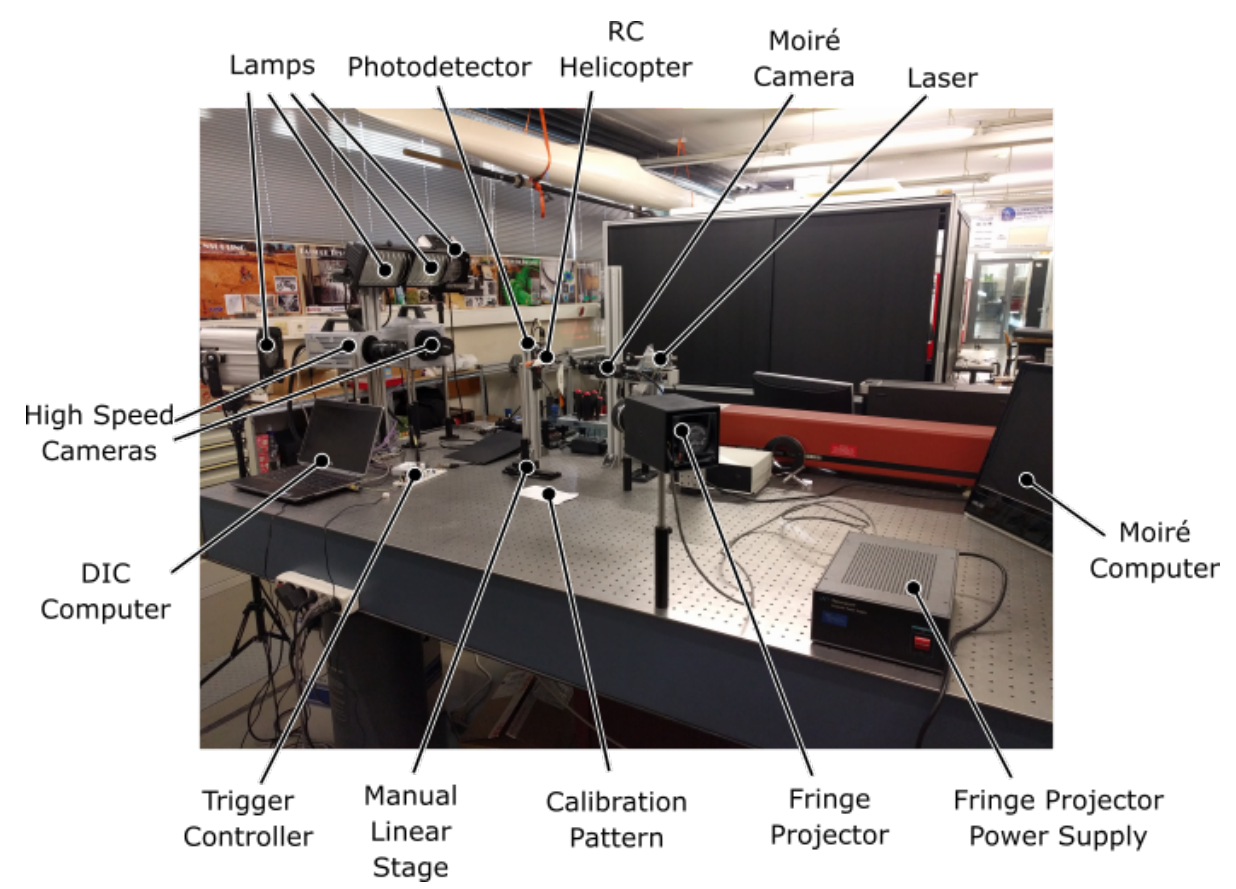

Figure 5: Experimental setup used for validation

were calculated as:

$$
\vec{u}=\left[\begin{array}{l}
0.0305 \\
0.2586 \\
0.9655
\end{array}\right], x_{0}=\left[\begin{array}{c}
-103.0181 \\
0.9453 \\
0
\end{array}\right]
$$

The angle between the two references was also calculated as $\alpha_{\text {ref }}=10.8424^{\circ}$ considering the top edge as the target. This consideration was used consistently as well for the remaining reported situations.

From the calculated value for $\vec{u}$, it is possible to compare the orientation of both coordinate systems, Figure 6 . The position of $\vec{u}$ between the $Z$ and $Y$ axis helps to explain the errors that were highlighted in previous work [17], where the displacements along the rotation axis would contribute to these two axes' representations.

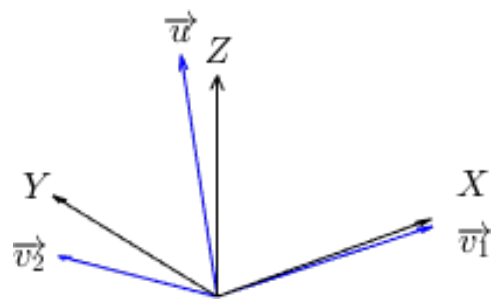

Figure 6: Comparison of the orientation of the original $(X, Y, Z)$ and the new $\left(\overrightarrow{v_{1}}, \overrightarrow{v_{2}}, \vec{u}\right)$ coordinate systems

For each of the imposed displacements, the best-fit angle was calculated by 
registration of the top edges, obtaining a correction angle for each:

$$
\begin{aligned}
& \alpha_{2 \mathrm{~mm}}=-7.1568^{\circ} \\
& \alpha_{4 \mathrm{~mm}}=-7.1796^{\circ} \\
& \alpha_{5 \mathrm{~mm}}=-7.1948^{\circ} \\
& \alpha_{7 \mathrm{~mm}}=-7.2219^{\circ}
\end{aligned}
$$

Finally, applying the Algorithm in Sections 2.2 and 2.3, the points were corrected and the displacements in $\left(\overrightarrow{v_{1}}, \overrightarrow{v_{2}}, \vec{u}\right)$ were calculated.

Figure 7 shows the resulting displacements for the $5 \mathrm{~mm}$ imposed displacement case. For each of the cases, a value in a constant position close to the application point was extracted (red cross in Figure 7c), having obtained:

$$
\begin{aligned}
& \Delta \vec{u}_{2 \mathrm{~mm}}=-2.0512 \\
& \Delta \vec{u}_{4 \mathrm{~mm}}=-3.9550 \\
& \Delta \vec{u}_{5 \mathrm{~mm}}=-5.0473 \\
& \Delta \vec{u}_{7 \mathrm{~mm}}=-7.0962
\end{aligned}
$$

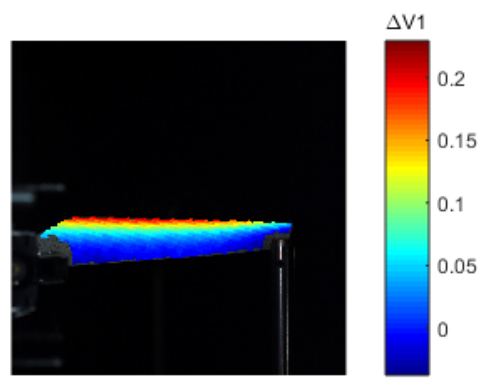

(a) Displacements along $\overrightarrow{v_{1}}$

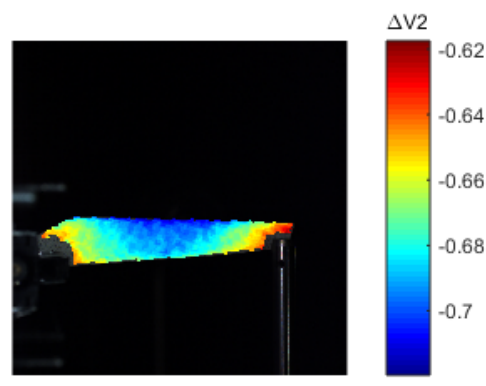

(b) Displacements along $\overrightarrow{v_{2}}$

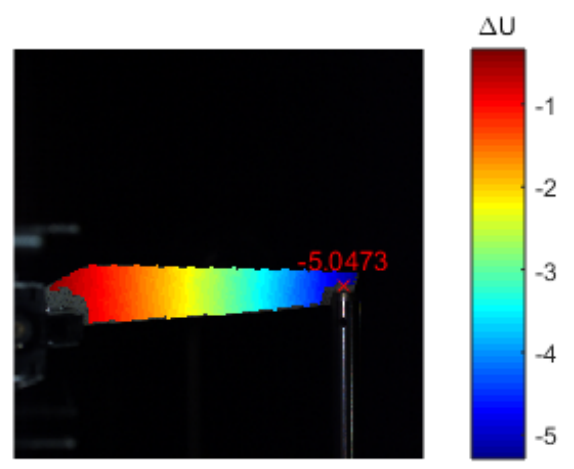

(c) Displacements along $\vec{u}$

Figure 7: Displacements in the $\left(\overrightarrow{v_{1}}, \overrightarrow{v_{2}}, \vec{u}\right)$ coordinate system for an imposed displacement of $5 \mathrm{~mm}$ 
As such, the errors for each situation were:

$$
\begin{aligned}
& \operatorname{Error}\left(\Delta \vec{u}_{2 \mathrm{~mm}}\right)=+0.0512 \mathrm{~mm}=+2.56 \% \\
& \operatorname{Error}\left(\Delta \vec{u}_{4 \mathrm{~mm}}\right)=-0.0450 \mathrm{~mm}=-1.12 \% \\
& \operatorname{Error}\left(\Delta \vec{u}_{5 \mathrm{~mm}}\right)=+0.0473 \mathrm{~mm}=+0.95 \% \\
& \operatorname{Error}\left(\Delta \vec{u}_{7 \mathrm{~mm}}\right)=+0.0962 \mathrm{~mm}=+1.37 \%
\end{aligned}
$$

Thus, the errors were less than $0.1 \mathrm{~mm}$, which is below $3 \%$. Considering that the movement was performed with resolution of a tenth of a millimetre, the obtained results closely match the expected result and, as such, help validate the developed methodology.

\section{Application Example}

As a final analysis, the developed methodology was applied to a dynamic situation. It was performed using the setup shown in Figure 1, and excluding Projection Moiré and the manual linear stage in Figure 5.

Before the actual dynamic measurements, the preparation phase again involved the acquisition of reference and calibration images. Afterwards, the trigger controller was configured for the situation at hand, the helicopter was started and one acquisition per rotation was performed. This generated 115 pairs of images at a speed of approximately $680 \mathrm{rpm}$. An exposure of $1 \mu \mathrm{s}$ was used, during which it is expected that the blade's tip moves around $0.013 \mathrm{~mm}$.

The used cameras allow for high framerates, $2000 \mathrm{fps}$ at full resolution. However, in this experiment, the framerate is not predefined as the acquisition of each image is performed asynchronously, with just one frame acquired for each rotation. If other slower cameras were used or if the rotation speed was too high, it would also be possible, for example, to acquire just one frame every two or more rotations.

The images were processed using VIC-3D 2012 from U.S.A.'s Correlated Solutions and the data was exported to MATLAB for processing. First, the axis and centre of rotation were calculated, having obtained:

$$
\vec{u}=\left[\begin{array}{l}
0.0099 \\
0.2274 \\
0.9738
\end{array}\right], x_{0}=\left[\begin{array}{c}
-103.5792 \\
-6.8325 \\
0
\end{array}\right], \alpha_{\text {ref }}=17.6933^{\circ}
$$

It should be noted that, since this experiment was not performed simultaneously with the experimental validation, small differences in the axis and centre of rotation are expected due to slight orientation changes in the cameras' positioning and other factors.

Afterwards, for each of the 115 revolutions, the point clouds can be corrected and the displacements in the $\left(\overrightarrow{v_{1}}, \overrightarrow{v_{2}}, \vec{u}\right)$ coordinate system can be calculated. Since the results are very similar among these frames, a single one's, the $15^{\text {th }}$, are presented.

In this case, the calculated best-fit angle was small, but still significant:

$$
\alpha=0.8106^{\circ}
$$




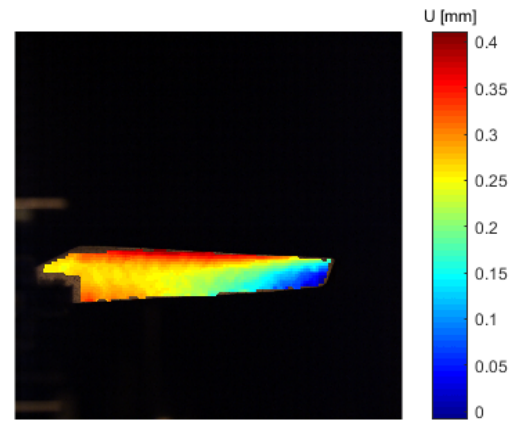

(a) Displacement along $\overrightarrow{v_{1}}$

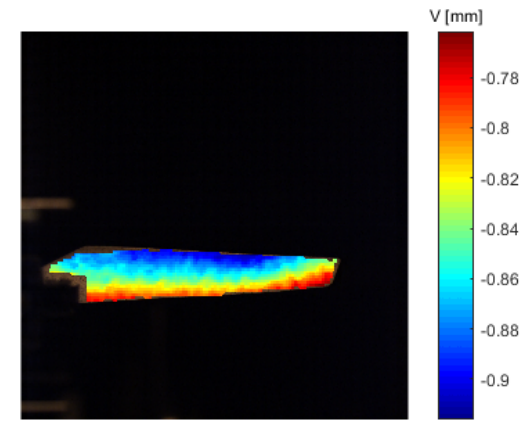

(b) Displacement along $\overrightarrow{v_{2}}$

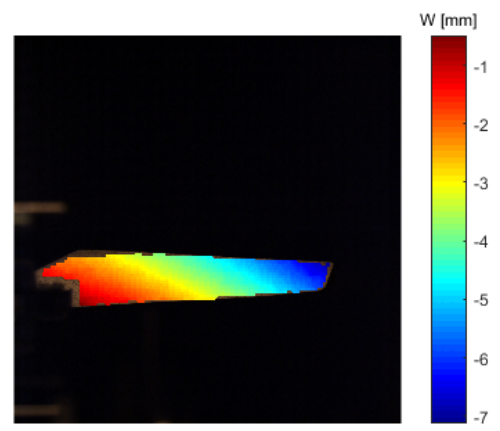

(c) Displacement along $\vec{u}$

Figure 8: Displacements in the $\left(\overrightarrow{v_{1}}, \overrightarrow{v_{2}}, \vec{u}\right)$ coordinate system using the developed algorithm

After correcting the point clouds using the calculated values, it was finally possible to calculate the displacements in the $\left(\overrightarrow{v_{1}}, \overrightarrow{v_{2}}, \vec{u}\right)$ coordinate system.

The obtained displacements are shown in Figure 8, while the uncorrected ones, i.e. the output from VIC-3D, are shown in Figure 9.

By comparing both sets of data, it is possible to see that the main improvements are:

- For the displacements along $\overrightarrow{v_{1}}$ and $X$, it is possible to see slight changes in the pattern, mainly due to the rotation correction, since the new coordinate system was created to maintain $\overrightarrow{v_{1}}$ and $X$ close and, therefore, their difference is small;

- The displacements along $\overrightarrow{v_{2}}$ and $Y$ are noticeably different, mainly because the displacements along the $\vec{u}$ rotation axis contributed to both $Y$ and $Z$ displacements and, since their magnitude is larger than the ones along $\overrightarrow{v_{2}}$, they mask their presence;

- Finally, the displacements along $\vec{u}$ and $Z$ are very similar, showing mostly changes in magnitude due to the new coordinate system, with the first having larger displacements due to a better alignment. For example, the minimum values are $-6.7490 \mathrm{~mm}$ along $Z$ and $-7.1205 \mathrm{~mm}$ along $\vec{u}$. 


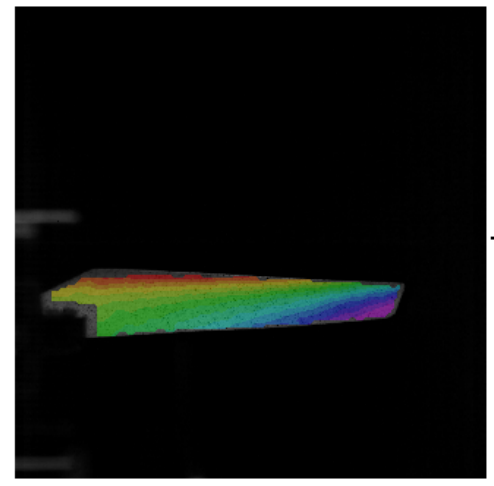

(a) Displacement along $\mathrm{X}$
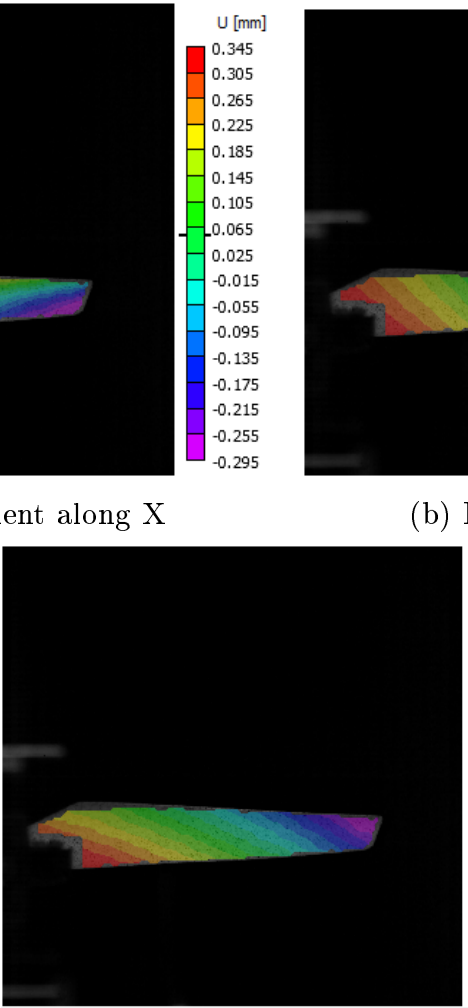

(c) Displacement along Z

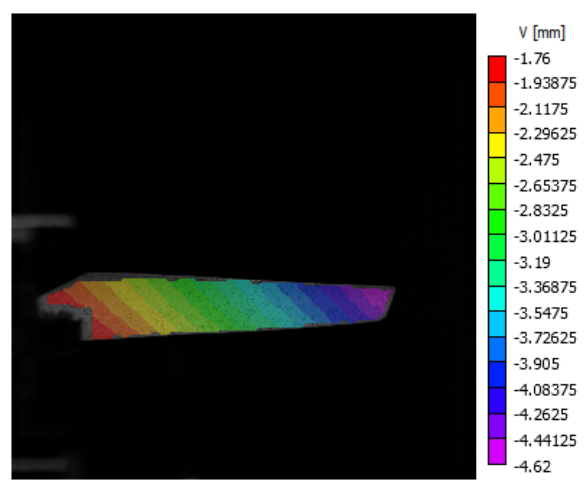

(b) Displacement along Y

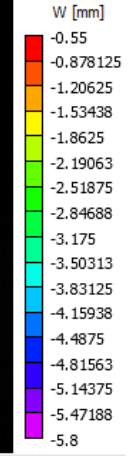

$-5.8$

Figure 9: Displacements in the $(X, Y, Z)$ coordinate system calculated by VIC-3D software

It was also possible to notice that the average displacements for $\overrightarrow{v_{1}}$ and $\overrightarrow{v_{2}}$ were 0.2443 and -0.8482 . This difference drew attention to the existence of gaps between the helicopter's blade and axis assembly of this order of magnitude.

\section{Conclusions}

A methodology for data correction through detection of the rotation axis was developed and validated. This enables the correction of two factors: misplacement of the reference image, causing over-rotation in the images, and the misalignment of the coordinate system and the rotation axis, which resulted in unintuitive representations.

Through the proposed methodology, by comparing two different reference situations, it was possible to obtain a centre and an axis of rotation for an $\mathrm{RC}$ helicopter's blade that were close to the expected values. Afterwards, it was possible to use this knowledge to calculate the best-fit rotation angle between the dynamic load situation and one of the references. This enabled both the removal of the effects of an over-rotation between these situations and the calculation of the displacements in a more appropriate coordinate system, i.e., one that contains the rotation axis and two perpendicular axes. Combining these two factors, it was possible to obtain an accurate measurement of the deformation state of an RC helicopter's blade under rotation. 
The new methodology was also experimentally validated by statically imposing known displacements and measuring the results, having obtained values that are very close to the imposed ones.

\section{Acknowledgements}

Pedro J. Sousa gratefully acknowledges the FCT ("Fundação para a Ciência e a Tecnologia") for the funding of the PhD scholarship SFRH/BD/129398/2017.

Dr. Moreira acknowledges POPH - QREN-Tipologia 4.2 - Promotion of scientific employment funded by the ESF and MCTES.

The authors also gratefully acknowledge the funding of Project NORTE01-0145-FEDER-000022 - SciTech - Science and Technology for Competitive and Sustainable Industries, co-financed by "Programa Operacional Regional do Norte" (NORTE2020) through "Fundo Europeu de Desenvolvimento Regional" (FEDER).

\section{References}

[1] I. Bucher, D. J. Ewins, Modal analysis and testing of rotating structures, Philosophical Transactions of the Royal Society of London. Series A: Mathematical, Physical and Engineering Sciences 359 (1778) (2001) 6196. doi:10.1098/rsta.2000.0714.

[2] J. Winstroth, J. R. Seume, Wind Turbine Rotor Blade Monitoring using Digital Image Correlation: Assessment on a Scaled Model, in: 32nd ASME Wind Energy Symposium, American Institute of Aeronautics and Astronautics, 2014. doi:doi:10.2514/6.2014-139610.2514/6.2014-1396. URL http://dx.doi.org/10.2514/6.2014-1396

[3] F. Boden, K. Bodensiek, B. Stasicki, Application of image pattern correlation for non-intrusive deformation measurements of fast rotating objects on aircrafts, Vol. 7522, 2009, pp. 75222S-75222S-10. URL http://dx.doi.org/10.1117/12.852703

[4] M. Ozbek, D. J. Rixen, Operational modal analysis of a $2.5 \mathrm{MW}$ wind turbine using optical measurement techniques and strain gauges, Wind Energy 16 (3) (2013) 367-381. doi:10.1002/we.1493.

URL http:https://doi.org/10.1002/we.1493

[5] F. Hild, S. Roux, Digital Image Correlation: from Displacement Measurement to Identification of Elastic Properties - a Review, Strain 42 (2) (2006) 69-80. doi:10.1111/j.1475-1305.2006.00258.x. URL http:https://doi.org/10.1111/j.1475-1305.2006.00258.x

[6] B. Stasicki, F. Boden, Application of high-speed videography for in-flight deformation measurements of aircraft propellers, Vol. 7126, 2008, pp. $712604-712612$.

URL http://dx.doi.org/10.1117/12.822046

[7] X. Zi, S. Geng, S. Zhao, F. Shu, Measurement of short shaft power based on a digital speckle correlation method, Measurement Science and Technology 26 (4) (2015) 45001. 
[8] S. Rizo-Patron, J. Sirohi, Operational Modal Analysis of a Helicopter Rotor Blade Using Digital Image Correlation, Experimental Mechanics 57 (3) (2017) 367-375. doi:10.1007/s11340-016-0230-6.

[9] J. Winstroth, L. Schoen, B. Ernst, J. R. Seume, Wind turbine rotor blade monitoring using digital image correlation: a comparison to aeroelastic simulations of a multi-megawatt wind turbine, Journal of Physics: Conference Series 524 (1) (2014) 12064.

URL http://stacks .iop.org/1742-6596/524/i=1/a=012064

[10] M. N. Helfrick, P. Pingle, C. Niezrecki, P. Avitabile, Optical Non-contacting Vibration Measurement of Rotating Turbine Blades (2009).

[11] C. Warren, C. Niezrecki, P. Avitabile, Optical Non-contacting Vibration Measurement of Rotating Turbine Blades II, in: Proceedings of the IMAC-XXVIII, 2010. doi:10.1007/978-1-4419-9716-6.

URL http://link.springer.com/10.1007/978-1-4419-9716$6\{\%\}$ Cnhttp: //www . springerlink. com/index/10.1007/978-1-4419$9716-6$

[12] J. Sirohi, M. S. Lawson, Measurement of helicopter rotor blade deformation using digital image correlation, Optical Engineering 51 (4) (2012) 4360343608. doi:10.1117/1.0E.51.4.043603.

URL http://dx.doi.org/10.1117/1.0E.51.4.043603

[13] J. Sicard, J. Sirohi, Measurement of the deformation of an extremely flexible rotor blade using digital image correlation, Measurement Science and Technology 24 (6) (2013) 65203.

URL http://stacks.iop.org/0957-0233/24/i=6/a=065203

[14] S. Rizo-Patron, J. Sirohi, Operational Modal Analysis of a Helicopter Rotor Blade Using Digital Image Correlation, Experimental Mechanics (2016) 19doi: 10.1007/s11340-016-0230-6.

URL http://dx.doi.org/10.1007/s11340-016-0230-6

[15] S. S. Rizo-Patron, J. Sirohi, Operational Modal Analysis of a Rotating Cantilever Beam Using High-Speed Digital Image Correlation, in: 57th AIAA/ASCE/AHS/ASC Structures, Structural Dynamics, and Materials Conference, American Institute of Aeronautics and Astronautics, 2016. doi:doi:10.2514/6.2016-195710.2514/6.2016-1957. URL http://dx.doi.org/10.2514/6.2016-1957

[16] P. J. Sousa, F. Barros, P. J. Tavares, P. M. Moreira, Displacement measurement and shape acquisition of an RC helicopter blade using Digital Image Correlation, Procedia Structural Integrity 5 (2017) 1253-1259. doi: $10.1016 / j$.prostr.2017.07.097.

URL http://linkinghub.elsevier.com/retrieve/pii/ S2452321617302093

[17] P. J. Sousa, F. Barros, P. J. Tavares, P. M. Moreira, Digital image correlation displacement measurement of a rotating RC helicopter blade, Engineering Failure Analysis 90 (March) (2018) 371-379. doi: $10.1016 / j$.engfailanal.2018.04.005. 
URL

http://linkinghub.elsevier.com/retrieve/pii/

S1350630717313328

[18] J. Winstroth, J. R. Seume, Wind turbine rotor blade monitoring using digital image correlation: 3d simulation of the experimental setup, in: European Wind Energy Association - EWEA 2014, no. March 2014, Barcelona, 2014.

[19] Correlated Solutions, Vic-3D 2010 Reference Manual (2010).

[20] G. mbH, GOM Correlate Professional V8 SR1 Manual Basic - Inspection - 3D Testing (2015).

[21] M. A. Sutton, J. J. Orteu, H. Schreier, Image correlation for shape, motion and deformation measurements: basic concepts, theory and applications, Springer Science \& Business Media, 2009.

[22] Correlated Solutions, Digital Image Correlation.

URL http://correlatedsolutions.com/digital-image-correlation/

[23] C. W. Spoor, F. E. Veldpaus, Rigid body motion calculated from spatial co-ordinates of markers, Journal of Biomechanics 13 (4) (1980) 391-393. doi : 10.1016/0021-9290(80) 90020-2.

[24] F. E. Veldpaus, H. J. Woltring, L. J. M. G. Dortmans, A least-squares algorithm for the equiform transformation from spatial marker co-ordinates, Journal of Biomechanics 21 (1) (1988) 45-54. doi:10.1016/00219290 (88) $90190-X$.

[25] W. C. Rose, J. G. Richards, Estimating Body Segment Motion by Tracking Markers, Journal of Chemical Information and Modeling 53 (9) (2012) 1689-1699. arXiv:arXiv:1011.1669v3, doi:10.1017/ CB09781107415324.004.

[26] E. W. Weisstein, Rotation Matrix.

URL http://mathworld.wolfram.com/RotationMatrix.html

[27] MathWorks, Rotation matrix for rotations around z-axis - MATLAB rotz. URL https://www . mathworks.com/help/phased/ref/rotz.html

[28] MathWorks, Minimum norm least-squares solution to linear equation MATLAB lsqminnorm (2017).

URL https://www . mathworks.com/help/matlab/ref/lsqminnorm.html

[29] The GSL Team, Linear Algebra - GSL 2.4 documentation (2017). URL https://www.gnu.org/software/gsl/doc/html/linalg.html

[30] MathWorks, Moore-Penrose pseudoinverse - MATLAB pinv. URL https://www.mathworks.com/help/matlab/ref/pinv.html

[31] E. W. Weisstein, Point-Line Distance-3-Dimensional. URL http://mathworld.wolfram.com/Point-LineDistance3Dimensional.html 
[32] E. W. Weisstein, Projection.

URL http://mathworld.wolfram.com/Projection.html

[33] G. Cloud, Optical Methods in Experimental Mechanics: Part 22: Projection Moiré, Experimental Techniques 30 (4) (2006) 15-18. doi:10.1111/ j.1747-1567.2006.00056.x. 